\title{
MONOID RINGS THAT ARE FIRS
}

\author{
ANDREU PITARCH
}

\begin{abstract}
It is well-known that the monoid ring of the free product of a free group and a free monoid over a skew field is a fir. We give a proof of this fact that is more direct than the proof in the literature.
\end{abstract}

History. In essence, the result is due to P. M. Cohn [3],[4] who showed that over a division ring the monoid ring of a free monoid is a fir, and that the monoid ring described in the abstract is a semifir, from which it follows fairly easily that it is a fir since it is hereditary. There are four other proofs in the literature: one due to $J$. Lewin [7] using Schreier rewriting techniques; one due to G. M. Bergman [1] using free products; one due to P. M. Cohn and W. Dicks $[6]$ using localization in firs; and one due to R. W. Wong [8] using only the normal form in a free group.

Definitions and notation. Let $X, Y$ be two disjoint sets. Let $M$ be the free product of the free monoid on $X$ and the free group on $Y$. Let $G$ be the free group on $X \cup Y$. We view $M \subseteq G$.

Each $c \in G$ has a unique normal form $c=c_{1} c_{2} \ldots c_{n}$, such that $n \geq 0$, $c_{i} \in X \cup X^{-1} \cup Y \cup Y^{-1}$ and $c_{i} c_{i+1} \neq 1$; in this case we write $l(c)=n$. We remark that if $c \in M$ then each $c_{i} \in X \cup Y \cup Y^{-1}$.

Let $<$ be any well-order of $X \cup X^{-1} \cup Y \cup Y^{-1}$. We extend this to the lengthlexicographic well-order $<$ of $\mathrm{G}$, that is, if $c=c_{1} c_{2} \ldots c_{n}, d=d_{1} d_{2} \ldots d_{m}$ are elements of $G$ in normal form then we write $c<d$ to mean that either $l(c)<l(d)$, or $l(c)=l(d)$ and for some $j$ such that $I \leq j \leq n$ we have $c_{1}=d_{1}, \ldots, c_{j-1}=d_{j-1}$ and $c_{j}<d_{j}$.

Let $K$ be a skew field. We write $K[M]$ for the monoid ring, and view it as a subring of the group ring $K[G]$. An element $x$ of $K[G]$ has a unique expression in the form $x=\sum_{c \in G} x(c) c$ where $x: G \rightarrow K$ is a function which takes the value 0 except for a finite number of elements of $G$. We shall treat interchangeably the elements of $K[G]$ and their corresponding functions. We define Supp $x=\{c \in G \mid x(c) \neq 0\}$.

We have functions

$$
l: K[G] \rightarrow\{-\infty\} \cup N, \text { such that } l(x)=\max \{l(c) \mid c \in \operatorname{Supp} x\}
$$


$\operatorname{deg}: K[G] \rightarrow\{-\infty\} \cup G$, such that $\operatorname{deg}(x)=\max \{c \mid c \in \operatorname{Supp} x\}$, where we understand $\max =-\infty$.

It is easy to see that $l(x)=l(\operatorname{deg}(x))$ for all $x \neq 0$, and $\operatorname{deg}(K[M])=$ $\{-\infty\} \cup M$. We call $l(x)$ the length of $x$.

Let $x \in K[G]$ and $a \in G$. If $l(a c)=l(a)+l(c)$ for all $c \in \operatorname{Supp} x$, we shall write $a \cdot x$ to mean $a x$; otherwise $a \cdot x$ is undefined.

Let $I$ be a left ideal of $K[M]$ and $x \in I$. We shall say that $x$ is isolated in $I$ if, whenever $x=\sum_{i=1}^{n} r_{i} x_{i}, r_{i} \in K[M], x_{i} \in I$, then $\operatorname{deg}\left(x_{j}\right) \geq \operatorname{deg}(x)$ for some $j$ with $1 \leq j \leq n$.

Let $a \in G$. We set

$$
a \cdot G=\{b \in G \mid b=a \cdot c \text { for some } c \in G\} .
$$

We define the right transduction with respect to $a$ to be the function

$$
[]^{a}: G \longrightarrow\{0\} \cup G
$$

such that $[a \cdot b]^{a}=b$ for all $a \cdot b \in a \cdot G$ and $[d]^{a}=0$ for all $d \in G \backslash a \cdot G$. This extends by linearity to $K[G]$, i.e. $\left\{\sum_{c \in G} x(c) c\right]^{a}=\sum_{c \in G} x(c)[c]^{a}$.

It is clear that $[K[M]]^{a} \subseteq K[M]$ for all $a \in G$.

Observe that if $a \in G, b \in G \backslash\{1\}$ such that $a b=a \cdot b$ and $x \in K[G]$ then $[a x]^{a \cdot b}=[x]^{b}$.

The result. First we state a lemma.

Lemma. Let $x, y$ be elements of $K[G]$ with $x \neq 0$, and $a$ be a nontrivial element of $G$, so $a=b \cdot c$ for some $c \in X \cup Y \cup X^{-1} \cup Y^{-1}$. Then

(i) $[y x]^{a}=[y]^{a} x-c^{-1} \cdot \sum_{d \in a \cdot G} y(d)[x]^{\left(c \cdot[d]^{a}\right)^{-1}}+\sum_{d \in G \backslash a \cdot G} y(d)[d x]^{a}$.

(ii) $l\left([y x]^{a}-[y]^{a} x+c^{-1} \cdot y(a)[x]^{c^{-1}}\right)<l(x)$.

(iii) $l\left(c[y x]^{a}-c[y]^{a} x-c \cdot y(b)[x\}^{c}\right)<l(x)$.

Proof: Since all the expressions involved are $K$-linear in $x$ and $y$, it suffices to consider this case where $x, y \in G$. There are three cases.

CASE 1. $y \in a \cdot G$

Here $y=a \cdot y^{\prime}$ where $y^{\prime}=[y]^{a}$ and (i) reduces to

$$
[y x]^{a}=[y]^{a} x-c^{-1} \cdot[x]^{\left(c \cdot y^{\prime}\right)^{-1}}
$$

which can be rewritten as

$$
\left[c y^{\prime} x\right]^{c}=y^{\prime} x-c^{-1} \cdot[x]^{\left(c \cdot y^{\prime}\right)^{-1}} .
$$


CASE 1A. $x \in y^{\prime-1} \cdot c^{-1} \cdot G$. Then $c y^{\prime} x \in G \backslash c \cdot G$ and both sides of (i') reduces to 0 .

Since $c y^{\prime} x=[x]^{\left(c \cdot y^{\prime}\right)^{-1}}$, (iii) holds in this case.

To see (ii) be consider the cases $y^{\prime} \neq 1$ and $y^{\prime}=1$. If $y^{\prime} \neq 1$, then $y \neq a$ and so $l\left(c^{-1} \cdot[x]^{\left(c \cdot y^{\prime}\right)^{-1}}\right)<l(x)$. If $y^{\prime}=1$ then $y=a$ and so $[y]^{a} x=x=c^{-1}[x]^{c^{-1}}$, (ii) holds in this case.

CASE 1B. $x \in G \backslash y^{\prime-1} \cdot c^{-1} \cdot G$. Then $c y^{\prime} x \in c \cdot G$ and both sides of (i') reduces to $y^{\prime} x$.

Moreover $y \neq b$, thus (iii) reduces to $l(0)<l(x)$.

To see (ii) consider the cases $y^{\prime} \neq 1$ and $y^{\prime}=1$. If $y^{\prime} \neq 1$, then $y \neq a$, and (ii) reduces to $l(0)<l(x)$. If $y^{\prime}=1$, then $[x]^{c^{-1}}=0$ and (ii) reduces to $l(0)<l(x)$.

CASE 2, $y=b$

Here $[y x]^{a}=[b x]^{a}=[x]^{c}$, which gives (i) in this case, (ii) reduces to $l\left([x]^{c}\right)<$ $l(x)$ and (iii) reduces to $l(0)<l(x)$.

CASE 3. $y \in G \backslash a \cdot G$ and $y \neq b$

Here (i) reduces to the triviality $[y x]^{a}=[y x]^{a}$.

CASE 3A. $[y x]^{a}=0$. In this case (ii) and (iii) reduces to $l(0)<l(x)$.

CASE 3B. $[y x]^{a} \neq 0$. Since $y(a)=y(b)=0$ and $[y]^{a}=0$, (ii) and (iii) reduce to $l\left([y x]^{a}\right)<l(x)$ and $l\left(c[y x]^{a}\right)<l(x)$ respectively. So in this case it suffices to show that $\left.l(\mid y x]^{a}\right)<l(x)-1$. Here $y x=a \cdot d$ where $d=|y x|^{a}$. It is easy to see that there exist $e, y^{\prime}, x^{\prime} \in G$ such that $y=y^{\prime} \cdot e, x=e^{-1} \cdot x^{\prime}$ and $y^{\prime} \cdot x^{\prime}=a \cdot d$. Since $y \in G \backslash a \cdot G$ it follows that $y^{\prime} \in G \backslash a \cdot G$. Hence there exists $f \in G \backslash\{1\}$ such that $a=y^{\prime} \cdot f$, so $y^{\prime} \cdot x^{\prime}=a \cdot d=y^{\prime} \cdot f \cdot d$ and $x^{\prime}=f \cdot d$. Thus $l\left(x^{\prime}\right)-l(f)=l(d)=l\left([y x]^{a}\right)$. Since $l(x)=l\left(x^{\prime}\right)+l(e)$ we see $l\left([y x]^{a}\right)=l(x)-l(e)-l(f)$. Thus it suffices to show that $l(e)+l(f) \geq 2$. We know $l(f) \geq 1$. If $e=1$ then $y=y^{\prime}$ and $a=y \cdot f$, but $y \notin a \cdot G$ and $y \neq b$, thus $l(f) \geq 2$.

Theorem (Lewin [7], Cohn [3]). $K[M]$ is a fir.

Proof: Let $I$ be a left ideal of $K[M]$. We set

$$
I^{*}=\{x \in I \mid x \text { is isolated in } I\}
$$

and introduce an equivalence relation $\sim$ in $I^{*}$ by defining $x \sim y$ if $\operatorname{deg}(x)=$ $\operatorname{deg}(y)$, for all $x, y \in I^{*}$.

Let $B$ be a complete set of representatives of the $\sim$-classes in $I^{*}$. We shall show that $B$ is a left $K[M]$-basis of $I$.

To see that $I$ is generated by $B$, let us suppose that it is not true, and choose $z \in I \backslash K[M] B$ of rninimum possible degree.

If $z$ is not isolated in $I$ then exists an expression $z=\sum r_{i} z_{i}$ with $r_{i} \in$ $K[M], z_{i} \in I$ and $\operatorname{deg}\left(z_{i}\right)<\operatorname{deg}(z)$. By the minimality of the degree of $z$, $z_{i} \in K[M] B$; hence $z \in K[M] B$, a contradiction. 
If $z$ is isolated in $I$ then exists $x \in B$ with $\operatorname{deg}(z)=\operatorname{deg}(x)$, so there exists a unique $r \in K$ such that $\operatorname{deg}(z-r x)<\operatorname{deg}(z)$. Now $z-r x \in I$ and by the minimality of the degree of $z, z-r x \in K[M] B$; hence $z \in K[M] B$, a contradiction.

These contradictions show that $I$ is generated by $B$, and it remains to show that $B$ is left $K[M]$-independent. Suppose then that it is dependent, so there exist distinct $x_{1}, x_{2} \ldots x_{n}$ in $B$ and nonzero $y_{1}, y_{2}, \ldots y_{n}$ in $K[M]$ such that $\sum_{i=1}^{n} y_{i} x_{i}=0$.

Since the $x_{i}$ are distinct elements of $B$, we may assume that

$$
\operatorname{deg}\left(x_{n}\right)>\operatorname{deg}\left(x_{n-1}\right)>\ldots>\operatorname{deg}\left(x_{1}\right) .
$$

We shall use right transduction with respect to the element $a=\operatorname{deg}\left(y_{n}\right)$. Since $x_{n}$ is isolated in $I$, it follows that $a \neq 1$, so $a=b \cdot c$ for some $c \in$ $X \cup Y \cup Y^{-1}$.

Consider the element

$$
W=\sum_{i=1}^{n}\left[y_{i} x_{i}\right]^{\alpha}-\sum_{i=1}^{n}\left[y_{i}\right]^{a} x_{i}=-\sum_{i=1}^{n}\left[y_{i}\right]^{a} x_{i}=-y_{n}(a) x_{n}-\sum_{i=1}^{n-1}\left[y_{i}\right]^{a} x_{i},
$$

it is clear that $W \in I$. Since $x_{n}$ is isolated in $I$, we see $\operatorname{deg}(W) \geq \operatorname{deg}\left(x_{n}\right)$. By part (i) of the Lemma,

$$
W=\sum_{i=1}^{n}\left(-c^{-1} \cdot \sum_{d \in a \cdot G} y_{i}(d)\left[x_{i}\right]^{\left(c \cdot[d)^{a}\right)^{-1}}+\sum_{d \in G \backslash \alpha \cdot G} y_{i}(d)\left[d x_{i}\right]^{a}\right),
$$

and by part (ii) of the Lemma,

$$
l\left(W+c^{-1} \cdot \sum_{i=1}^{n} y_{i}(a)\left[x_{i}\right]^{c^{-1}}\right)<l\left(x_{n}\right) .
$$

Since $\operatorname{Supp}\left(y_{i}(a) c^{-1} \cdot\left[x_{i}\right]^{c^{-1}}\right) \subseteq \operatorname{Supp} x_{i}$ for all $i=1, \ldots, n$, and $\operatorname{deg}\left(x_{i}\right)<$ $\operatorname{deg}\left(x_{n}\right)$ for all $i=1, \ldots, n-1$, thus $\operatorname{deg}(W)=\operatorname{deg}\left(y_{n}(a) c^{-1} \cdot\left[x_{n}\right]^{c^{-i}}\right)=$ $\operatorname{deg}\left(x_{n}\right) \in c^{-1} \cdot G$. In particular $c^{-1} \in M$, and there exists a unique $r \in K$ such that $\operatorname{deg}\left(x_{n}-r W\right)<\operatorname{deg}\left(x_{n}\right)$. Now from the equation $x_{n}=\left(x_{n}-r W\right)+$ $\left(r c^{-1}\right) c W$ and the fact $x_{n}$ is isolated in $I$ we see that $\operatorname{deg}(c W) \geq \operatorname{deg}\left(x_{n}\right)$. By part (i) of the Lemma,

$$
c W=\sum_{i=1}^{n}\left(-\sum_{d \in a \cdot G} y_{i}(d)\left[x_{i}\right]^{\left.(c \cdot d]^{a}\right)^{-1}}+c \cdot \sum_{d \in G \backslash a \cdot G} y_{i}(d)\left[d x_{i}\right]^{a}\right),
$$

and by part (iii) of the Lemma,

$$
l\left(c W-c \cdot \sum_{i=1}^{n} y_{i}(b)\left[x_{i}\right]^{c}\right)<l\left(x_{n}\right) .
$$


Since Supp $\left(y_{i}(b) c \cdot\left[x_{i}\right]^{c}\right) \subseteq \operatorname{Supp} x_{i}$ for all $i=1, \ldots, n$, and $\operatorname{deg}\left(x_{i}\right)<\operatorname{deg}\left(x_{n}\right)$ for all $i=1, \ldots, n-1$, thus $\operatorname{deg}\left(x_{n}\right)=\operatorname{deg}\left(y_{n}(b) c \cdot\left[x_{n}\right]^{c}\right) \in c \cdot G$, which contradicts the fact that $c \cdot G \cap c^{-1} \cdot G=\emptyset$.

Thus $B$ is a basis for $I$, and $I$ is free as left $K[M]$-module.

By the symmetry of the hypotheses, every right ideal is free as right module, so $K[M]$ is a fir.

Acknowledgments. I am grateful to Ferran Cedó and Warren Dicks for all their help in the preparation of this article.

\title{
References
}

1. G. M. Bergman, Modules over coproducts of rings, Trans. Amer. Math. Soc. 200 (1974), 1-32.

2. G. M. BERGMAN AND W. DICKS, Universal derivations and universal ring constructions, Pacific J. 79 (1978), 293-337.

3. P. M. Corn, On a generalization of the Euclidean algorithm, Proc. Cambridge Philos. Soc. 57 (1961), 18-30.

4. P. M. CoHN, On the free product of associative rings III, J. Algebra 8 (1968), 376-383.

5. P. M. Conn, "Free rings and their Relations," Second edition, London Math. Soc. Monograph 19, Academic Press, London, 1985.

6. P. M. Cohn AND W. DICks, Localization in semifirs II, J. London Math. Soc. (2) 13 (1976), 411-418.

7. J. LEWIN, Free modules over free algebras and free group algebras: The Schreier technique, Trans. Amer. Math. Soc. 145 (1969), 455-465.

8. Roman W. Wong, Free ideal monoid rings, J. Algebra 53 (1978), 21-35.

\author{
Departament de Matemàtiques \\ Facultat de Ciencies \\ Universitat Autònoma de Barcelona \\ 08193 Bellaterra (Barcelona) \\ SPAIN
}

Rebrt el 4 de Setembre de 1989 